\title{
Assessment of the incidence of accessory hepatic arteries: a literature review
}

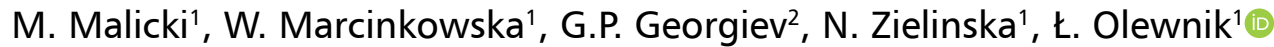 \\ ${ }^{1}$ Department of Anatomical Dissection and Donation, Medical University of Lodz, Poland \\ 2Department of Orthopaedics and Traumatology, University Hospital Queen Giovanna-ISUL, \\ Medical University of Sofia, Bulgaria
}

[Received: 31 May 2021; Accepted: 17 July 2021; Early publication date: 24 August 2021]

\begin{abstract}
Modern medicine is developing towards application of endovascular techniques such as trans-arterial hepatic chemoembolisation. They displace classic open procedures. However, their correct planning and performance depend on the knowledge pre-operative detection of hepatic arterial anatomical variations. The main abnormality that may generate complications during radiological and surgical procedures is occurrence of an accessory hepatic artery. In the present study we propose our own classification of the variability observed in the vessels based on cases reported in the literature. It analyses more types of variations as compared to previous trials. A great advantage of this study is also a description of different pathological and frequently life-threatening conditions associated with hepatic arteries. This study is of value to medical practitioners, e.g. surgeons. (Folia Morphol 2022; 81, 3: 533-543)
\end{abstract}

Key words: accessory hepatic artery, liver vascularisation, aneurysm, morphological variations, hepatic artery, new classification, coeliac trunk, superior mesenteric artery, left hepatic artery, right hepatic artery

\section{INTRODUCTION}

An uninterrupted blood supply is an essential condition for every organ to maintain life [40]. Anatomical variations of extrahepatic blood vessels are a common occurrence [37]. In the most dominant anatomical pattern the common hepatic artery (CHA) originates from the coeliac trunk (CT) [19]. Past the branch-off point, CHA runs forwards and laterally, reaching the superior surface of the duodenum [45]. Then it divides into the gastroduodenal artery (GDA) and the proper hepatic artery (PHA). GDA feeds into the pylorus and the proximal part of the duodenum [2]. PHA runs between the layers of the lesser omentum, within the hepatoduodenal ligament, then reaching the hepatic hilum [2]. It is located there posteriorly to the portal vein (PV) [25]. This vessel divides into the left hepatic artery (LHA) and the right hepatic artery (RHA) [19]. They supply the right and left lobes of the liver, respectively [2].

The liver is the largest internal organ in the human body. It receives approximately $25 \%$ of total cardiac output at rest. Interestingly, the liver has dual blood supply [2]. Hepatic arteries feed this organ with highly oxygenated blood [35]. Nevertheless, it is only up to $30 \%$ of the total blood supply. The remaining $70 \%$ is provided by PV [2].

We can define an accessory hepatic artery (aHA) as an additional arterial vessel that supplies the liver. It may fulfil a supportive function only or replace basic arteries supplying the organ [37]. Moreover,

Address for correspondence: Ł. Olewnik, DPT, PhD, Ass. Prof., Department of Anatomical Dissection and Donation, Medical University of Lodz, ul. Żeligowskiego 7/9, 90-136 Łódź, Poland, e-mail: lukasz.olewnik@umed.lodz.pl

This article is available in open access under Creative Common Attribution-Non-Commercial-No Derivatives 4.0 International (CC BY-NC-ND 4.0) license, allowing to download articles and share them with others as long as they credit the authors and the publisher, but without permission to change them in any way or use them commercially. 
these vessels are divided into left and right depending on which lobe of the liver they reach. The most common variations of such vessels are an accessory right hepatic artery (aRHA) arising from the superior mesenteric artery (SMA) and an accessory left hepatic artery (aLHA) originating from the left gastric artery (LGA) [22].

Hepatic arterial vascularisation may be related to serious disorders. The most serious ones are aneurysm and pseudoaneurysm. It is well-known that a ruptured aneurysm is a life-threatening condition [1]. It has been also reported that an aneurysm developing from an aHA is rather a rare condition [42]. Impairments such as stenosis and thrombosis of hepatic arteries are frequent complications following liver transplantation. When left untreated, they can be dangerous for the patient's life [13, 26, 29]. Interestingly, arterioportal shunts which are a pathologic anastomosis between the hepatic artery and PV may occur $[4,13]$.

The aim of this study is to systematise the current knowledge on occurrence of aHAs and their variations. We also focus on clinical syndromes related to arterial hepatic vascularisation. Different diagnostic techniques are also presented. The analysed information is crucial for proper performance of surgical procedures such as liver transplantation or pancreaticoduodenectomy. Therefore, the present study is particularly valuable for radiologists and surgeons.

\section{EMBRYOLOGY}

Knowledge of embryology is necessary to comprehend the origin of arterial variations in the liver [11]. Based on angiogenesis, hepatic vascular development progresses during embryogenesis in order to form a complex network of hepatic vessels [43]. The embryonic liver in the $5^{\text {th }}$ week of gestation contains three major veins, i.e. the umbilical veins, the vitelline veins and the cardinal veins. The vitelline veins are the major efferent vessels which transport the deoxygenated blood to the inferior vena cava. The left vitelline vein then involutes and blood is directed to the right vitelline vein, which enlarges and forms the hepato-cardiac part of the inferior vena cava [5]. Branches of the vitelline veins also form the hepatic vein. In contrast, the umbilical vein is involved in the development of the afferent hepatic venous system. However, it mostly degenerates. Only a part of the left umbilical vein remains and connects to the right vitelline vein via a ductus venosus. As the umbilical vein collapses, it is replaced by the portal vein as the
Table 1. Hiatt's classification of hepatic arterial anatomic variants

\begin{tabular}{lc}
\hline Type & Description \\
\hline I & CHA from CT (normal pattern) \\
II & Accessory or replaced LHA from LGA \\
III & Accessory or replaced RHA from SMA \\
IV & Co-occurrence of accessory or replaced LHA from \\
& LGA and accessory or replaced RHA from SMA \\
V & CHA from SMA \\
VI & CHA from the aorta \\
\hline
\end{tabular}

Abbreviations - see text

main afferent vein $[5,43,44]$. The formation of the hepatic artery occurs later than the development of the veins [43]. The hepatic artery arises from the aorta and passes through the portal vein in the parenchyma and it gradually expands. After birth, there is closure of the umbilical arteries, veins and ductus venosus and they form the medial umbilical ligament, the ligamentum teres and the ligamentum venosum. Postnatal morphology involves blood supply to the liver via the hepatic artery and the portal vein and its outflow through the hepatic veins $[5,43,44]$. It can be hypothesized that arterial variations such as aLHA and aRHA are caused by differences in embryonic development and are remainder of the left and right embryonic hepatic arteries [11].

\section{CLASSIFICATION OF ACCESSORY HEPATIC ARTERIES}

The anatomy and various variants of the hepatic arteries have been thoroughly classified and characterised in the literature. In 1966 Michels [31] published the classification system in which he identified ten types of hepatic artery variations [31]. The classification was based on 200 dissections. It contained a division into accessory and replaced hepatic arteries. The types of variants included differences in the supply of specific hepatic arteries. The classification, based on 1000 cases [22], was updated in 1994 by Hiatt et al. [22], who described six types (Table 1) taking into account the presence of accessory or replacement vessels to reduce the division proposed by Michels [31].

Several scientists have also investigated the frequency of the different types. Table 2 presents the investigation trials that analysed the greatest number of cases. Michels's classification is based on dissections [31]. The study conducted by Hiatt et al. [22] involved observations of patients who underwent 
Table 2. Summary of variants of accessory hepatic arteries presented in the literature

\begin{tabular}{lccccc}
\hline Type & $\begin{array}{c}\text { Hiatt et al. [22] } \\
(\mathbf{n}=\mathbf{1 0 0 0})\end{array}$ & $\begin{array}{c}\text { Michels et al. [31] } \\
(\mathbf{n = 2 0 0})\end{array}$ & $\begin{array}{c}\text { Hanif et al. [20] } \\
(\mathbf{n}=\mathbf{1 0 0 0})\end{array}$ & $\begin{array}{c}\text { Koops et al. [25] } \\
(\mathbf{n}=\mathbf{6 0 4})\end{array}$ & $\begin{array}{c}\text { Abdullah et al. [3] } \\
(\mathbf{n}=\mathbf{9 3 2})\end{array}$ \\
\hline Normal pattern & $75.7 \%$ & $55 \%$ & $64.4 \%$ & $79.1 \%$ & $68.1 \%$ \\
aLHA from LGA & $9.7 \%$ & $18 \%$ & $13.5 \%$ & $3 \%$ & $8.1 \%$ \\
aRHA from SMA & $10.6 \%$ & $18 \%$ & $12.1 \%$ & $11.9 \%$ & $10.2 \%$ \\
aLHA + aRHA & $2.3 \%$ & $4 \%$ & $7.3 \%$ & $1.3 \%$ & $6.4 \%$ \\
Others & $1.7 \%$ & $5 \%$ & $2.7 \%$ & $4.7 \%$ & $7.2 \%$ \\
\hline
\end{tabular}

Abbreviations - see text

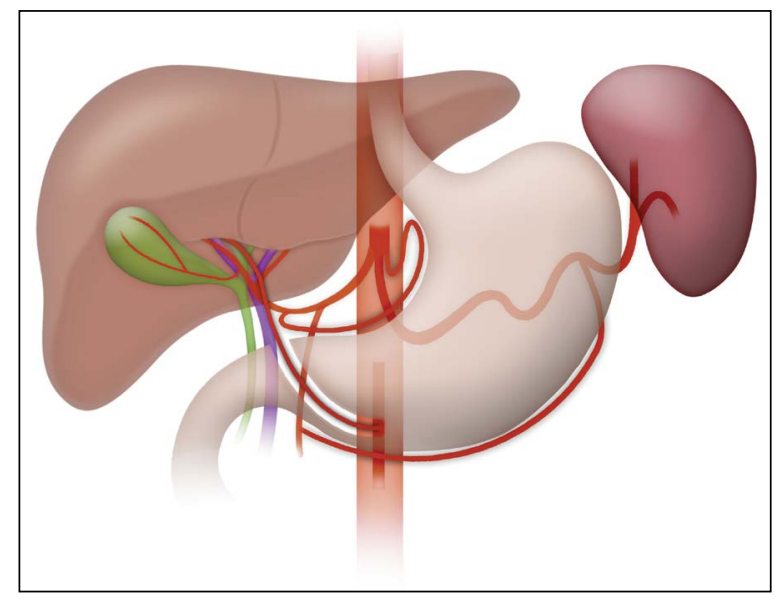

Figure 1. Type III, subtype A - accessory right hepatic artery arising from superior mesenteric artery.

liver harvesting for orthotopic transplantation [22]. A similar method was applied by Abdullah et al. [3], Hanif et al. [20] and Koops et al. [25] who focused on abdominal angiography in their investigations [20, 25]. Moreover, Hanif et al. [20] examined only the population of Pakistan [20].

Based on the results demonstrated in Table 2, normal anatomy is evidently the most commonly occurring one. Among the variants of aHA, aRHA arising from SMA is observed most frequently. A slightly less commonly occurring one is aLHA branching off from the LGA. The other types are rare.

The occurrence of aRHA branching from SMA (Fig. 1) is widely reported in the literature [12, 14, 28, $32,48]$. This is definitely the most common variant of aRHA categorised as type III in the Hiatt's classification [22]. Li et al. [28] described the case of a 67-year-old female patient who was diagnosed with intrahepatic bile duct cancer [28]. A computed tomography scan revealed aRHA arising from SMA. The accessory vessel passed through the right posterior side of the portal vein, then it was wrapped from the posterior to anterior side to its right branch and it distributed

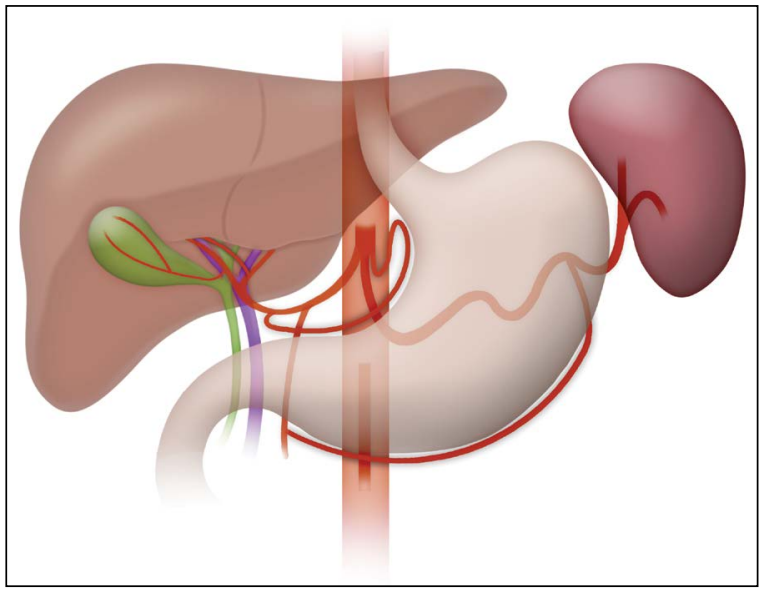

Figure 2. Type II, subtype $\mathrm{C}$ - accessory right hepatic artery branching from left hepatic artery.

blood in the right anterior part of the liver, supplying segments V and VIII [28]. The same variation was also reported by $\mathrm{Yu}$ et al. [48]. In a 47-year-old female, aRHA originating from SMA was detected during liver transplantation surgery. The vessel supplied the posterior segment of the liver graft [48].

The literature also reports a rare retroportal course of aRHA from LHA [30] (Fig. 2). This variation was identified in the liver of a 52-year-old male. We can observe the arterial vessel located distally to the bifurcation of the portal vein which supplies the right posterior segments VI-VII [30].

Also, aRHA may branch off from GDA (Fig. 3). This case was discovered by Yamashita et al. [47] in a 41 -year-old female patient with gallbladder cancer [47]. Three-dimensional angiography revealed that aRHA was running in front of the common bile duct into the anterior segment of the liver. Interestingly, the cholecystic artery branched off it. Thus, aRHA supplied blood both into the liver and the gallbladder [47].

In the literature there are also reports on two capabilities of aRHA branching from the CT $[8,34]$. 


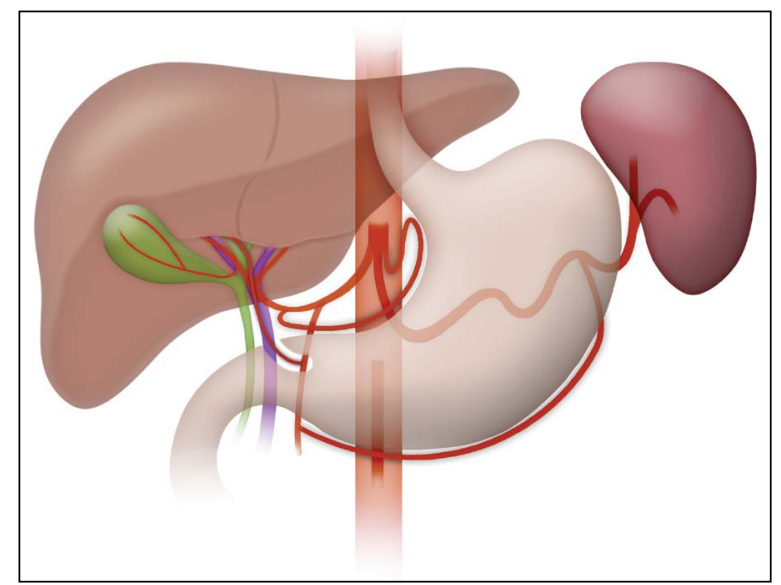

Figure 3. Type II, subtype D - accessory right hepatic artery originating from gastroduodenal artery.

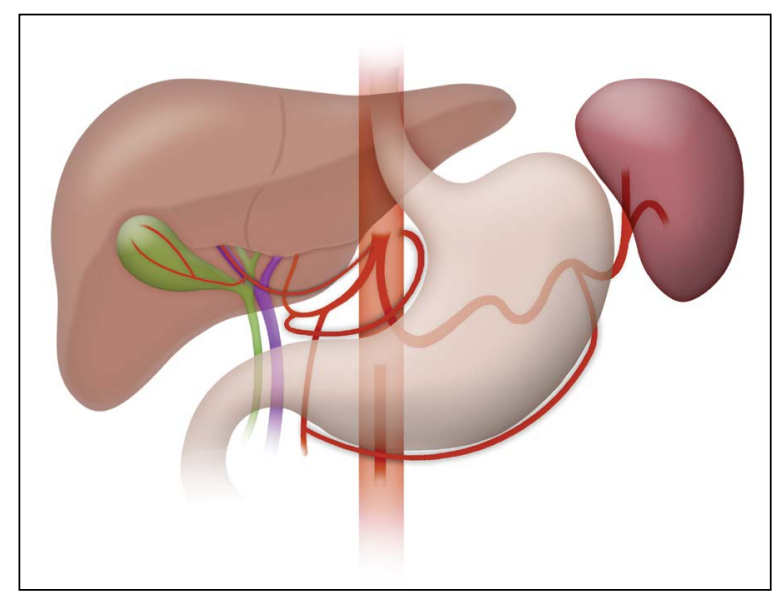

Figure 4. Type I, subtype A - accessory right hepatic artery branching from trifurcation variation of coeliac trunk.

One of them is the case of trifurcation variation of $C T$ that contains aRHA (Fig. 4). CHA, the splenic artery (SA) and aRHA originate from the common arterial trunk and LGA arises directly from the abdominal aorta [34]. The second one is the case of a four-branch trunk (Fig. 5). The CT gives off three basic arteries, i.e. CHA, SA, LGA, and an additional fourth branch, i.e. aRHA [8].

Another unusual phenomenon is the branching of aRHA from LGA (Fig. 6). Such an anatomic variation was discovered in a female who died at the age of 92 years. An aHA arising from LGA and reaching the left lobe of the liver is definitely more frequent. In the described case, LGA branches off CT. After running a short course, it divides into three branches. Two of them are abnormal large gastric arteries and the third one is aRHA. In this study the course of this vessel

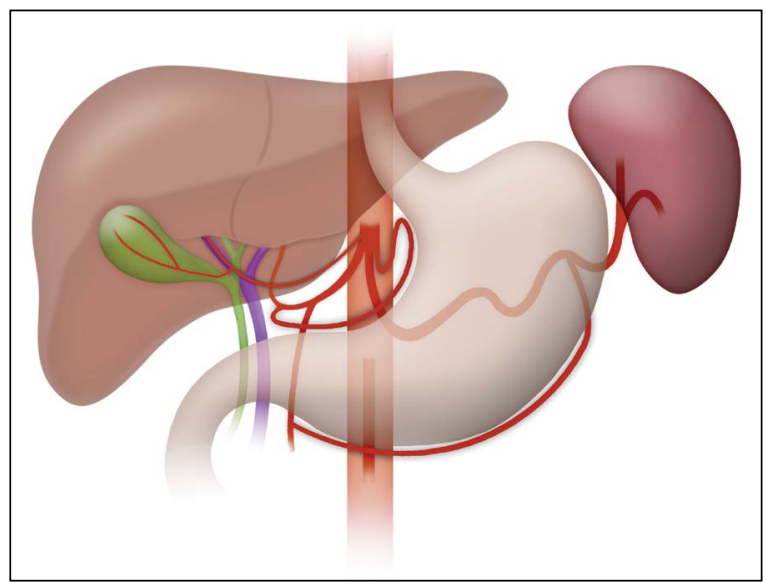

Figure 5. Type I, subtype B - accessory right hepatic artery originating from four-branch coeliac trunk.

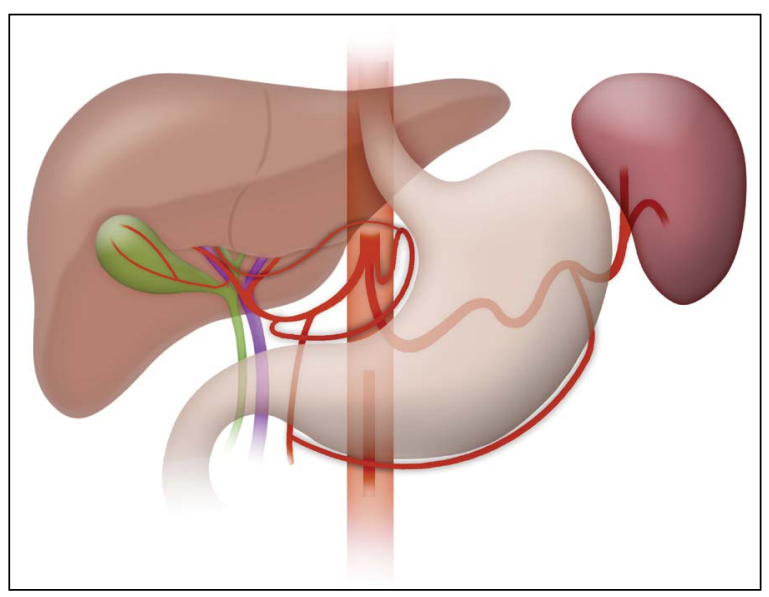

Figure 6. Type I, subtype C - accessory right hepatic artery arising from left gastric artery.

was carefully explored. It has been confirmed that it supplies the right lobe of the liver [37].

Also, the presence of aRHA arising from the right renal artery (RRA) (Fig. 7), was identified on renal computerised tomographic angiogram by Darsan et al. [10]. There were two reported cases of subjects with the anatomical variation. However, the courses of accessory vessels were different. In the first case a 46-year-old female had aRHA that branched from RRA near its origin from the abdominal aorta. Then, it passed aloft behind the inferior vena cava and ramified in the right lobe of the liver. In the second case involving a 50-year-old male, aRHA branched from RRA close to the renal hilum. Then, it passed along the right crus of diaphragm. The aRHA also supplied the right lobe of the liver [10].

In the literature there are also cases presenting aRHA that originates from CHA (Fig. 8). The case of 


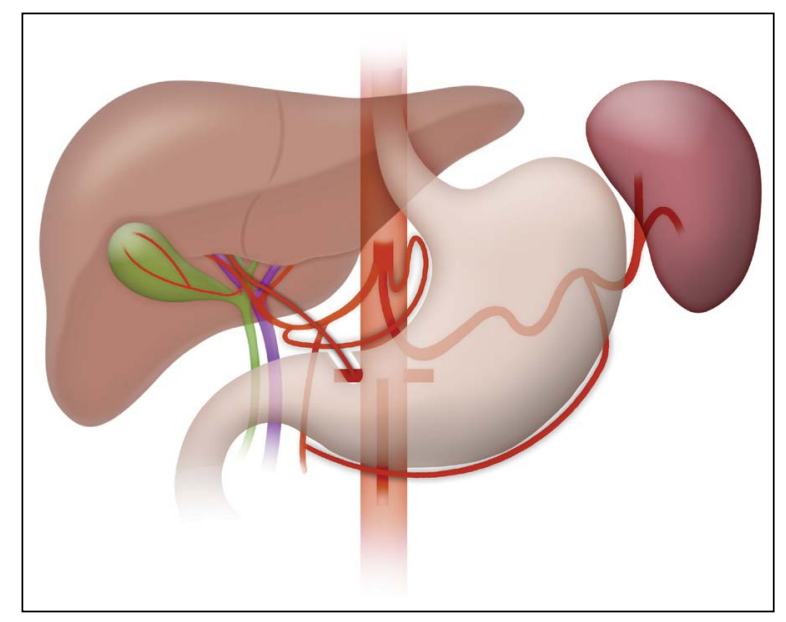

Figure 7. Type IV — accessory right hepatic artery originating from right renal artery.

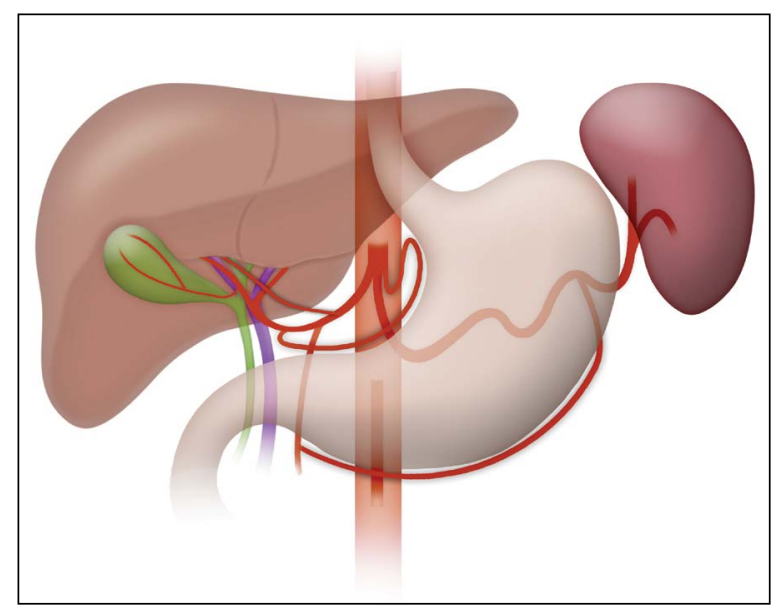

Figure 8. Type II, subtype A - accessory right hepatic artery originating from common hepatic artery.

a 65-year-old Caucasian female shows this kind of an abnormality. The CHA arose from the coeliac trunk, which is a typical condition. However, after a course of $9 \mathrm{~mm}$ it branched off aRHA. The vessel passed behind the portal vein and supplied the right lobe of the liver [40].

The occurrence of aRHA arising from the SA (Fig. 9) was also reported in the literature by Caruso et al. [6]. In the case of a 67-year-old cadaveric organ donor, aRHA crossed downward and parallel to CHA, after branching from SA. Then it passed behind the gastroduodenal artery and the common bile duct. Ultimately, aRHA ran along the right side of the portal vein and entered the right lobe of the liver [6].

There also occur cases of aRHA branching off directly from the periphery of the abdominal aorta (Fig. 10) [12].

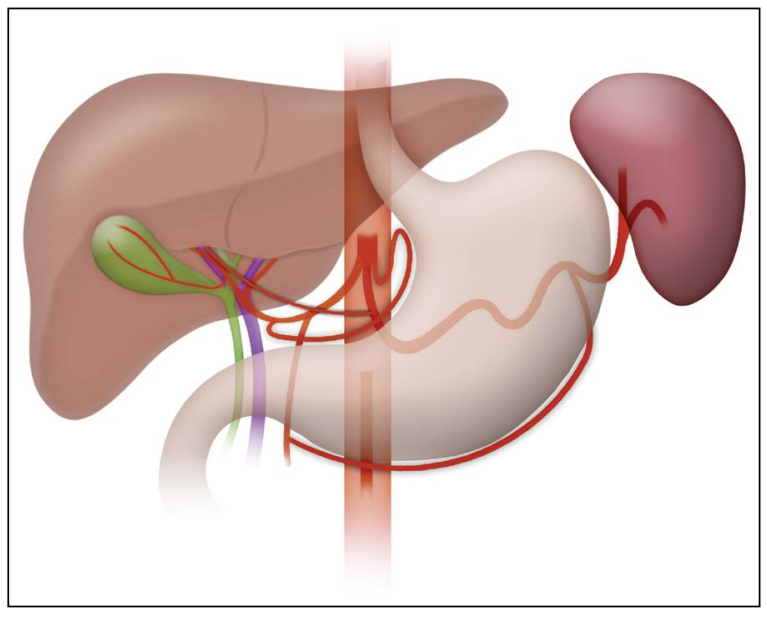

Figure 9. Type I, subtype E - accessory right hepatic artery branching from splenic artery.

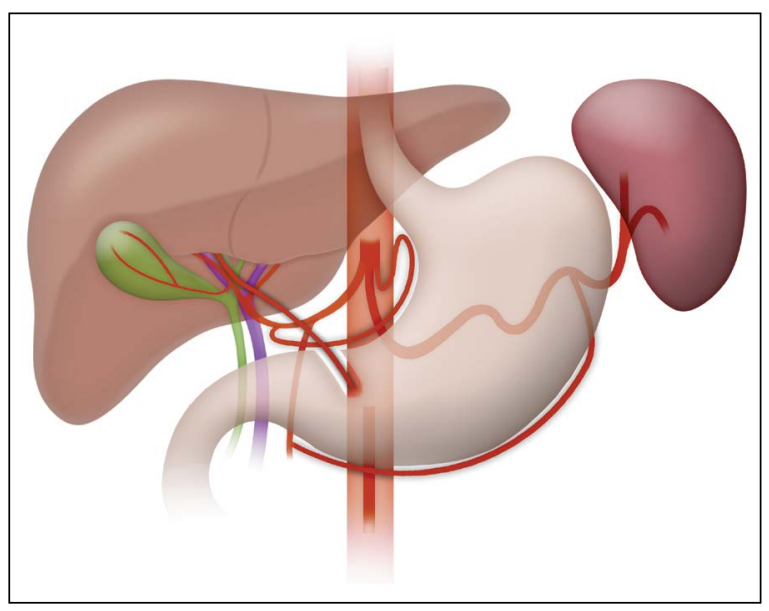

Figure 10. Type V - accessory right hepatic artery arising from abdominal aorta.

The occurrence of an aLHA is less prevalent than the aRHA [35]. In the literature, the most commonly described kind of variation is aLHA arising from LGA $[12,14,20,38]$ (Fig. 11). It is the type II according to the Hiatt's classification [22]. During dissection of the cadaver of a 94-year-old man, such an abnormality was identified. The aLHA originated from the branching area of LGA and then it ran upwards supplying blood to the left liver lobe [38].

In some individuals, aLHA branches off from SMA (Fig. 12) [12, 20].

A rare occurrence of aLHA leading from CHA (Fig. 13) has also been reported. In the case of a 55-year-old male, aLHA was extending opposite to GDA and ran upwards and medially to PHA [35].

Another interesting phenomenon are patients in whom both aHA occur simultaneously in addition to 


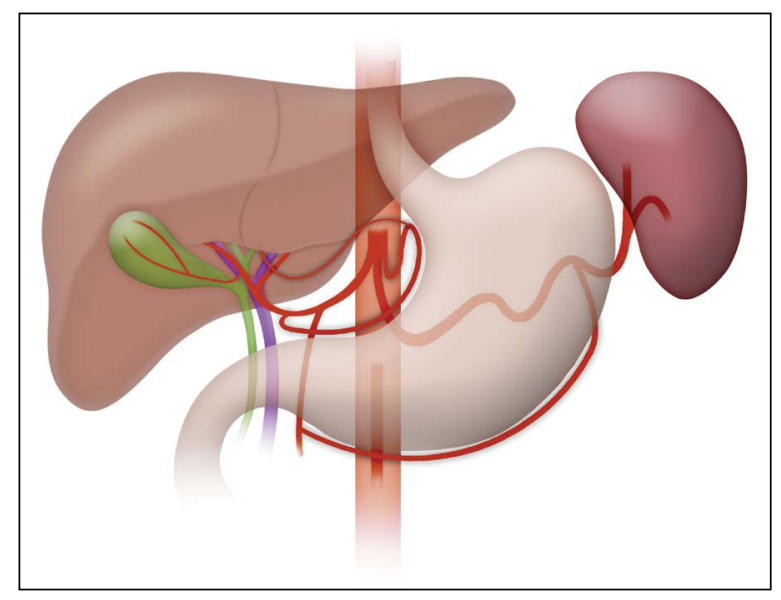

Figure 11. Type I, subtype D - accessory left hepatic artery branching from left gastric artery.

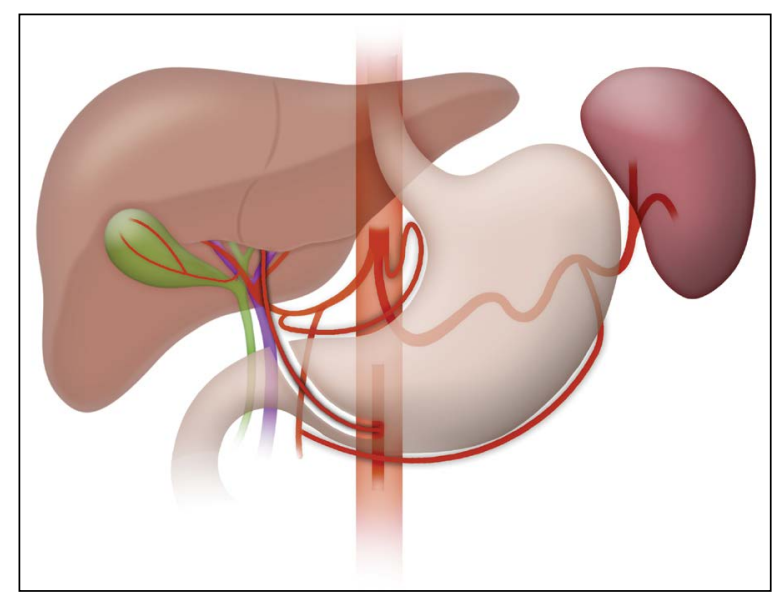

Figure 12. Type III, subtype B - accessory left hepatic artery branching from superior mesenteric artery.

the normal arterial supply (Fig. 14). In the case of an adult male cadaver, a triple arterial blood supply to the liver was observed. There were also reported cases of aLHA arising from the LGA and running upwards in the hepatogastric ligaments. At the same time, aRHA originated from SMA and ran behind the common bile duct in the hepatoduodenal ligament [11]. Such arterial anomalies result from differences in embryonic development. However, it may cause complications associated with diagnostic and surgical procedures in the epigastric region [11]. Similarly, we identified a case of a 67-year-old female with both aHA, which is an example of type IV in Hiatt's classification [22]. The aLHA arose from LGA, whereas the aRHA originated from SMA, crossed the right posterior portal vein and distributed blood to the right anterior region of the liver to supply segments V and VIII [28]. Another

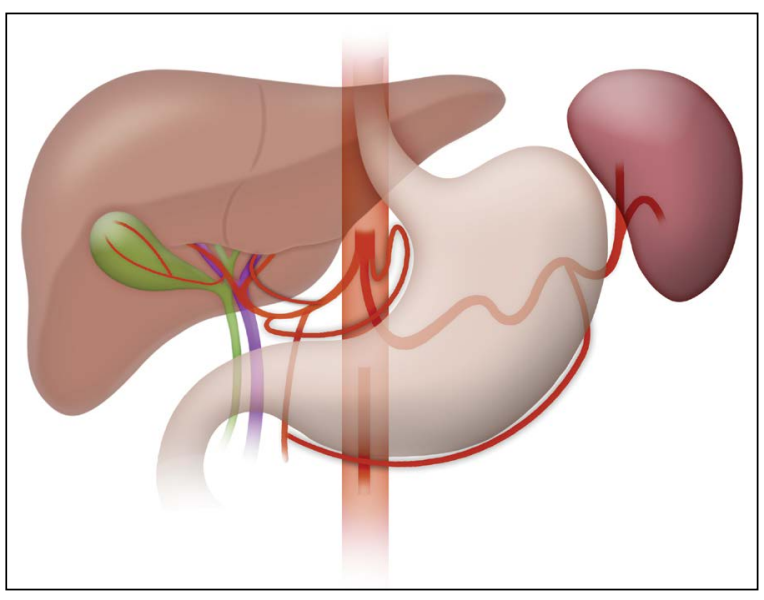

Figure 13. Type II, subtype B - accessory left hepatic artery arising from common hepatic artery.

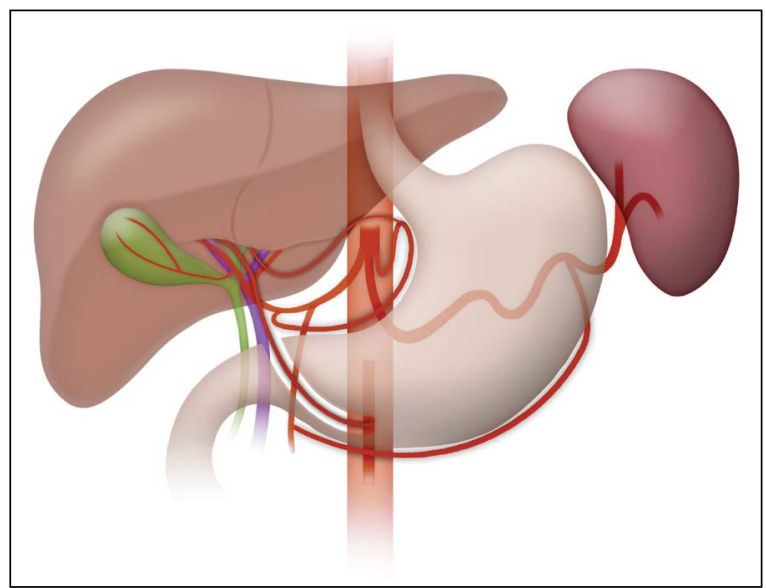

Figure 14. Type $\mathrm{VI}$ - co-occurrence of accessory right hepatic artery and accessory left hepatic artery.

unusually rare case of coexistence of aLHA and aRHA was noticed during a dissection of a human cadaver. The aLHA arose from CHA, supplying the stomach and the left liver lobe. The aRHA originated from GDA, running behind the cystic duct and then into the liver [24].

We proposed a new classification of aHA origins with a division into types and subtypes including all departures described in the literature (Table 3 ). This classification does not focus on the physiological significance of these arteries. Therefore, we do not divide them into accessory and replaced ones. Nevertheless, they are classified into right and left arteries depending on which part of the liver they supply. It is not a classification that serves didactic purposes; however, it is intended for clinicians in whose work liver vascularisation plays a key role. Therefore, it 
Table 3. New classification system based on the available literature by Malicki et al. (current study)

\begin{tabular}{ll}
\hline Type & Description \\
\hline I & $\begin{array}{l}\text { aHA from CT and its branches (except for } \\
\text { the branches associated with the CHA) }\end{array}$ \\
A & aRHA from trifurcation variation of CT \\
B & aRHA from four-branch CT \\
C & aRHA from LGA \\
D & aLHA from LGA \\
E & aRHA from SA \\
II & aHA from CHA and its branches \\
A & aRHA from CHA \\
B & aLHA from CHA \\
C & aRHA from LHA \\
D & aRHA from GDA \\
III & aHA from SMA \\
A & aRHA from SMA \\
B & aLHA from SMA \\
IV & aRHA from RRA \\
V & aRHA from AA \\
VI & Co-occurrence of aRHA and aLHA \\
\hline
\end{tabular}

Abbreviations - see text

avoids a complex division in order to create a clear and easy to read set of information. Our aim is to collect knowledge of all possible variations, which is essential in surgical specialties.

The classification includes six types in total. We distinguish type I in which aHA originates from CT and its branches, except for the branches associated with CHA. The arteries extending from CHA represent a separate category, i.e. type II to reduce the number of subtypes. This results in a classification that is readable for an analysis by researchers. Most of the types are related to aRHA. Only three of them refer to aLHA. However, the last type involves co-occurrence of both accessory arteries.

\section{CLINICAL SIGNIFICANCE OF THE ACCESSORY HEPATIC ARTERY}

Accessory hepatic arteries are frequently smaller than basic arterial hepatic vessels [40]. Nevertheless, they are functionally relevant [40]. We reviewed a case study that support this statement [15].

A 31-year-old male suffered in a motor vehicle collision. A CT scan of the chest, abdomen and pelvis demonstrated internal injuries. The most important one, in the context of the issue discussed in this article, was a pancreatic head haematoma which triggered interruption of arterial flow in the CHA. The examination also showed the presence of aLHA [15]. During surgical intervention it was observed that the liver parenchyma was still viable. The aLHA developed a rich net of collateral arterial vessels with the inferior phrenic artery. The additional source of liver blood supply was high significant in the case. Therefore, any major signs of acute liver ischaemia were not noticed. Despite extensive injuries, the patient suffered only temporary and limited ischaemic changes [15]. After one month following the accident, angiography, computed tomography and magnetic resonance imaging were performed. An enlargement of aLHA becoming the dominant arterial vessel was observed.

We can use this rare case as an example of "in vivo" presentation of clinical significance of additional hepatic artery occurrence. It has also been suggested that the well-known liver resilience to arterial ischaemia is a result of occurrence of other possible liver arterial flow sources [15]. It is obvious that a variable course of such vessels and functional significance cause complications during various abdominal procedures. Vessel damage may lead to dangerous bleeding. That is why it is so important to identify additional hepatic arteries preoperatively. It allows you to plan a correct procedure which often determines the success of a surgery [47].

However, it has been reported that the presence of aHA can be beneficial in the context of rare surgeries. Paloyo et al. [36] presented a case of a 64-year-old male. The patient underwent orthotopic liver transplantation. Interestingly, he suffered from portomesenteric vein thrombosis. It affected the portal vein, too. Such a pathological condition is a contraindication against liver transplantation. However, current achievements in surgical techniques can solve this problem. There are various options such as thromboendovenectomy, portocaval hemitransposition, renoportal anastomosis or arterialisation of PV. Thromboendovenectomy was performed as a first-choice procedure. Unfortunately, PV flow was still marginal. Another method had to be used in this case. Based on preoperative computed tomography the presence of aRHA branching from SMA was identified [36]. Thanks to this abnormal condition, PV arterialisation could be performed. The aRHA was accessible. Surgeons created end-to-end anastomosis between the additional hepatic artery and the recipient's PV. It was a highly effective and easy method 
of increasing portal flow. Doppler ultrasonography confirmed turbulent flow and increased pulsatility. Four years following liver transplantation, the patient had normal liver function. It is an extremely rare use of the presence of an aHA in order to facilitate a surgical procedure. Nevertheless, it shows that anatomical variations are not always an obstacle for surgeons only [36].

An aHA can be associated with various pathologic liver conditions. Vascular variants trigger many clinical syndromes [13]. In the present study, we focus on disorders related to liver arterial flow.

\section{THROMBOSIS}

Hepatic artery thrombosis frequently occurs as a complication following liver transplantation. Other surgeries such as Whipple procedure may also lead to this kind of thrombosis [13]. It is a life-threatening condition. It has been estimated that this disorder occurs in $2.5-9 \%$ of adults and in $9-15 \%$ of paediatric population [18].

There is no evidence in the literature that hepatic arterial abnormalities have an impact on the frequency of hepatic artery thrombosis incidence. However, it is clear that the presence of accessory arteries increase the number of anastomosis procedures that have to be performed during transplantation. It makes the technical aspect of the surgery more difficult. Despite this fact, it has been suggested that liver with vascular variations can be transplanted without increased risk [17]. Thrombosis complications require revascularisation. Unfortunately, this method often fails. It results in necessary re-transplantation [18]. Most often, early hepatic artery thrombosis is recognised during a routine postoperative ultrasound examination. This form of the disease is asymptomatic. If the thrombosis is not diagnosed in time, various complications can develop. The most significant one is liver parenchyma and duct ischaemia [21]. Interestingly, development of collateral arterial vessels can prevent liver failure. It has been reported that such patients are in good condition despite diagnosed irreversible hepatic artery thrombosis [17]. Prevention strategies can also play an important role. According to previous studies, implementation of acetylsalicylic acid therapy following liver transplantation may reduce the risk for occurrence of the disorder. The introduction of microvascular surgery is useful, too. An early diagnosis gives the chance for successful treatment.
Therefore, the use of Doppler ultrasonography may be essential [9].

\section{STENOSIS}

Hepatic artery stenosis (HAS) is one of the common complications following orthotopic liver transplantation. According to previous studies, the frequency of this disorder ranges from $3.5 \%$ to $11 \%$ $[26,29]$. We have not reviewed any papers containing a case report of the development of stenosis in relation to aHA. However, we speculate that this is a potential phenomenon as there are cases where this artery replaces the classic hepatic artery and becomes a significant provider of blood for the liver. Presumably, in a patient with aHA who has undergone liver transplantation, the vessel stenosis may occur as a classic post-operative complication following this type of surgery.

Most often, HAS is asymptomatic. However, it may result in hepatic artery thrombosis. It can also lead to liver ischaemic and biliary strictures [27, 29]. There are various well-known risk factors. The most relevant one, in the context of the analysed issue, is complex donor hepatic artery anatomy. It is also related to a prolonged operative time [27]. The choice of treatment method is a matter of discussion in the literature. Surgical management such as aorto-hepatic bypass or re-anastomosis following the resection of a stenotic segment is always an option for HAS. However, achievements in the recent years have made endovascular procedures a golden standard nowadays. Interventional radiology offers less invasive medical procedures and uses local anaesthesia only. Therefore, these techniques have advantages over conventional vascular surgery. Two main endovascular methods are percutaneous transluminal angioplasty and stenting. As for their efficiency, there are no significant differences between them. Nevertheless, using percutaneous transluminal angioplasty results in more common HAS recurrence than following stenting. That is why it was suggested that stenting could be a first-line treatment [29]. However, according to Pulitano et al. [41] study, endovascular treatment is given in the case of graft impairment or ischaemic biliary strictures. This kind of therapy is beneficial because it may reduce further graft damage. Endovascular methods should not be performed in asymptomatic patients or those diagnosed at a late stage of the disease [41]. 


\section{ARTERIOPORTAL SHUNTS}

It is the most common intrahepatic vascular shunt. We can also distinguish other types such as arteriovenous, portosystemic, portoportal or venovenous shunts. It is a kind of pathological anastomosis between the portal vein and the hepatic artery. It provides direct communication between these hepatic vessels. According to the laws of physics, oxygenated blood flows into the portal vein under high pressure. This process facilitates detection of this disorder by dynamic contrast-enhanced computed tomography or magnetic resonance imaging. The scan is characterised by an occurrence of a wedge-shaped geographic region. The arterioportal shunt is frequently a result of iatrogenic trauma such as liver biopsy. It may also be a concomitant disorder of haemangiomas or hepatocellular carcinoma. Most often such a shunt is asymptomatic $[4,13]$. In the literature, there is no evidence proving an association between the presence of arterioportal shunts and the occurrence of aHA. However, this condition of aHA is probably possible. Since direct communication between the classical artery and portal vein is possible, a similar anastomosis between the artery and portal vein may be created in patients with aHA.

\section{ANEURYSM}

We can define aneurysm as a pathological dilatation of a blood vessel. However, this basic definition is too general. Therefore, a better classification is required. The exact pathophysiology of aneurysm is still unclear. Nevertheless, many risk factors are wellknown [23]. Among them the most important ones are obesity, atherosclerosis, hypertension, peripheral artery disease, peptic ulcer disease. It has also been suggested that tobacco and alcohol consumption are closely related to aneurysm development [39].

Hepatic artery aneurysm (HAA) is a rare clinical condition. It has been estimated that HAA accounts for $20 \%$ of visceral aneurysms. It makes HAA the second most common visceral aneurysm following splenic artery aneurysm (SAA) [1].

Development of aHA aneurysm has been also reported. However, it is extremely rare [42]. Serena et al. [42] described a case of a 71-year-old Caucasian female who presented with aLHA mycotic pseudoaneurysm. Probably, the aetiological factor of this disorder was Clostridium difficile. Interestingly, the location of pseudoaneurysm precluded open surgery or percutaneous thrombin injection. Therefore, coil of this lesion was performed. Antibiotic therapy was also introduced [42].

Aneurysms have been divided into two main types. The first group are true aneurysms which have a wall consisting of all layers of vascular structure. They are related to arterial fibrodysplasia, polyarteritis nodosa, vasculitis, systemic lupus erythematosus. The second type are pseudoaneurysms that have a fibrous sac without the endothelium or vascular wall structure. The other term for this type is "false aneurysm". It develops as a result of blunt or penetrating injuries. However, the most common cause is iatrogenic trauma such as biopsy [4, 13, 23]. Interestingly, an infection may also result in this disorder. Mycotic aneurysms have been described in the literature [42]. True aneurysms are most often asymptomatic. Sometimes, patients may complain of abdominal pain. Therefore, these lesions are identified accidentally on imaging tests.

Hepatic artery aneurysm is a severe disorder. Rupture of HAA is a life-threatening condition. It can affect the peritoneal cavity, hepatobiliary tract, stomach or the duodenum. The frequency of HAA rupture ranges from $20 \%$ to $80 \%$ [1].

There are plenty of treatment strategies. An open repair procedure is surgical excision of an aneurysm and reconstruction of liver vascularisation. An arterial by-pass may be required [46]. Obviously, there are other less invasive techniques such as trans-arterial embolisation or covered stent placement [39]. There are no significant differences in overall mortality and post-operative complications between the traditional and endovascular treatment methods. However, the latter reduces the time of hospitalisation. Furthermore, it is beneficial for elderly patients since because of their age surgery may be contraindicated in some individuals. Therefore, endovascular treatment is recommended [16]. Currently, an aneurysm which has a diameter greater than $2 \mathrm{~cm}$ and non-atherosclerotic origin are main symptoms that predispose to intervention [33].

\section{HEPATOCELLULAR CARCINOMA}

Accessory hepatic arteries may also play an important role in cancer development. They can supply tumours. According to previous studies, this type of cancer has almost exclusively arterial vascularisation. The knowledge of liver vascular abnormalities is also essential for proper performance of chemoembolisation. It is a highly recommended treatment approach. 
The other options are only surgical hepatic resection or liver transplantation [7].

\section{CONCLUSIONS}

The functional importance and variable courses of aHA create a risk of complications during abdominal procedures. Their occurrence may also be associated with development of pathological conditions. Therefore, preoperative diagnosis of these arteries plays an important role. It enables radiologists and surgeons to obtain all significant information and properly plan the surgical procedure in advance.

\section{Conflict of interest: None declared}

\section{REFERENCES}

1. Abdallah FF, Serracino-Inglott F, Ananthakrishnan G. Giant hepatic aneurysm presenting with hematemesis successfully treated with an endovascular technique. Vasc Endovascular Surg. 2017; 51(5): 331-334, doi: 10.1177/1538574417707145, indexed in Pubmed: 28478708.

2. Abdel-Misih SRZ, Bloomston M. Liver anatomy. Surg Clin North Am. 2010; 90(4): 643-653, doi: 10.1016/j. suc.2010.04.017, indexed in Pubmed: 20637938.

3. Abdullah SS, Mabrut JY, Garbit V, et al. Anatomical variations of the hepatic artery: study of 932 cases in liver transplantation. Surg Radiol Anat. 2006; 28(5): 468-473, doi: 10.1007/s00276-006-0121-0, indexed in Pubmed: 16642277.

4. Albers BK, Khanna G. Vascular anomalies of the pediatric liver. Radiographics. 2019; 39(3): 842-856, doi: 10.1148/ rg.2019180146, indexed in Pubmed: 31059404.

5. Bhargava P, Vaidya S, Kolokythas O, et al. Pictorial review. Hepatic vascular shunts: embryology and imaging appearances. Br J Radiol. 2011; 84(1008): 1142-1152, doi: 10.1259/bjr/82649468, indexed in Pubmed: 22101582.

6. Caruso F, Dondossola D, Fornoni G, et al. Right hepatic artery from splenic artery: the four-leaf clover of hepatic surgery. Surg Radiol Anat. 2016; 38(7): 867-871, doi: 10.1007/s00276-016-1617-x, indexed in Pubmed: 26769020.

7. Cazejust J, Bessoud B, Colignon N, et al. Hepatocellular carcinoma vascularization: from the most common to the lesser known arteries. Diagn Interv Imaging. 2014; 95(1): 27-36, doi: 10.1016/j.diii.2013.04.015, indexed in Pubmed: 23978434

8. Chanasong R, Putiwat P, Roboon J, et al. Accessory hepatic artery arising from celiac trunk: an incidence in a Thai cadaver. Int J Morphol. 2014; 32(4): 1136-1139, doi: $10.4067 / \mathrm{s} 0717-95022014000400002$.

9. Compagnon $P$, Toso $C$. Selective retransplantation after late hepatic artery thrombosis. Transpl Int. 2019; 32(5): 470-472, doi: 10.1111/tri.13411, indexed in Pubmed: 30779232.

10. Darsan L, Vishal V, Cardoza F. Accessory right hepatic artery originating from proximal and distal right renal artery in two subjects. Indian J Urol. 2019; 35(4): 305-306, doi: 10.4103/iju.IJU_86_19, indexed in Pubmed: 31619873.

11. Dolenšek J. Triple arterial blood supply to the liver and double cystic arteries. Folia Morphol. 2017; 76(3): 523-526, doi: 10.5603/FM.a2017.0008, indexed in Pubmed: 28150275 .

12. Egorov VI, Yashina NI, Fedorov AV, et al. Celiaco-mesenterial arterial aberrations in patients undergoing extended pancreatic resections: correlation of $\mathrm{CT}$ angiography with findings at surgery. JOP. 2010; 11(4): 348-357, indexed in Pubmed: 20601809.

13. Elsayes KM, Shaaban AM, Rothan SM, et al. A comprehensive approach to hepatic vascular disease. Radiographics. 2017; 37(3): 813-836, doi: 10.1148/rg.2017160161, indexed in Pubmed: 28430541.

14. Erbay N, Raptopoulos V, Pomfret EA, et al. Living donor liver transplantation in adults: vascular variants important in surgical planning for donors and recipients. AJR Am J Roentgenol. 2003; 181(1): 109-114, doi: 10.2214/ ajr.181.1.1810109, indexed in Pubmed: 12818839.

15. Fernandes E, Pedrazzani C, Gerena M, et al. Traumatic common hepatic artery injury causing isolated right hepatic ischemia due to a left accessory artery. A case report. Int J Surg Case Rep. 2017; 39: 56-59, doi: 10.1016/j. ijscr.2017.07.061, indexed in Pubmed: 28806621.

16. Ferrara D, Giribono AM, Viviani E, et al. Endovascular management of a large hepatic artery aneurysm. Clin Ter. 2017; 168(3): e178-e180, doi: 10.7417/T.2017.2001, indexed in Pubmed: 28612892.

17. Fouzas I, Papanikolaou C, Katsanos G, et al. Hepatic artery anatomic variations and reconstruction in liver grafts procured in greece: the effect on hepatic artery thrombosis. Transplant Proc. 2019; 51(2): 416-420, doi: 10.1016/j.transproceed.2019.01.078, indexed in Pubmed: 30879555 .

18. Fouzas I, Sklavos A, Bismpa K, et al. Hepatic artery thrombosis after orthotopic liver transplantation: 3 patients with collateral formation and conservative treatment. Transplant Proc. 2012; 44(9): 2741-2744, doi: 10.1016/j. transproceed.2012.09.002, indexed in Pubmed: 23146510.

19. Garg S, Kumar KH, Sahni D, et al. Anatomy of the hepatic arteries and their extrahepatic branches in the human liver: a cadaveric study. Ann Anat. 2020; 227: 151409, doi: 10.1016/j.aanat.2019.07.010, indexed in Pubmed: 31400446.

20. Hanif F, Farooq U, Malik AA, et al. Hepatic artery variations in a sample of pakistani population. J Coll Physicians Surg Pak. 2020; 30(2): 187-191, doi: 10.29271/jcpsp.2020.02.187, indexed in Pubmed: 32036828.

21. Heaton ND. Hepatic artery thrombosis: conservative management or retransplantation? Liver Transpl. 2013; 19 (Suppl 2): S14-S16, doi: 10.1002/lt.23739, indexed in Pubmed: 24019107.

22. Hiatt JR, Gabbay J, Busuttil RW. Surgical anatomy of the hepatic arteries in 1000 cases. Ann Surg. 1994; 220(1): 50-52, doi: 10.1097/00000658-199407000-00008, indexed in Pubmed: 8024358.

23. Inston N, Mistry H, Gilbert J, et al. Aneurysms in vascular access: state of the art and future developments. J Vasc Access. 2017; 18(6): 464-472, doi: 10.5301/jva.5000828, indexed in Pubmed: 29099536. 
24. Jin W, Dong M, Pan J, et al. Rare combined variations of accessory left hepatic artery and accessory right hepatic artery: a case report and literature review. Surg Radiol Anat. 2020; 42(4): 443-447, doi: 10.1007/s00276-01902396-4, indexed in Pubmed: 31811353.

25. Koops A, Wojciechowski B, Broering DC, et al. Anatomic variations of the hepatic arteries in 604 selective celiac and superior mesenteric angiographies. Surg Radiol Anat. 2004; 26(3): 239-244, doi: 10.1007/s00276-004-0229-z, indexed in Pubmed: 14968265.

26. Lee DD, Paz-Fumagalli R, Croome KP, et al. Hepatic artery stenosis after liver transplant: Donation after cardiac death donor vs donation after brain death donor grafts. Clin Transplant. 2018; 32(11): e13413, doi: 10.1111/ctr.13413, indexed in Pubmed: 30240491.

27. Lee $\mathrm{D}$, Chung $\mathrm{BH}$, Heo $\mathrm{SH}$, et al. Case report of a large common hepatic artery aneurysm. Ann Vasc Surg. 2018; 52: 316.e11-316.e13, doi: 10.1016/j.avsg.2018.04.011, indexed in Pubmed: 29886208.

28. Li X, Zhang X, Lu Q, et al. An accessory right hepatic artery derived from the superior mesenteric artery for anterior right liver lobe supply: a case report. Surg Radiol Anat. 2019; 41(8): 969-971, doi: 10.1007/s00276-018-2173-3, indexed in Pubmed: 30580394.

29. Magand N, Coronado JL, Drevon H, et al. Primary angioplasty or stenting for hepatic artery stenosis treatment after liver transplantation. Clin Transplant. 2019; 33(12): e13729, doi: 10.1111/ctr.13729, indexed in Pubmed: 31630451.

30. Martins AC. Retroportal accessory right hepatic artery arising from the left hepatic artery: anatomy and surgical implications. HPB (Oxford). 2010; 12(9): 654-655, doi: 10.1111/j.1477-2574.2010.00204.x, indexed in Pubmed: 21158205.

31. Michels NA. Newer anatomy of the liver and its variant blood supply and collateral circulation. Am J Surg. 1966; 112(3): 337-347, doi: 10.1016/0002-9610(66)90201-7, indexed in Pubmed: 5917302.

32. Molmenti EP, Klein AS, Henry ML. Procurement of liver and pancreas allografts in donors with replaced/accessory right hepatic arteries. Transplantation. 2004; 78(5): 770-771, doi: 10.1097/01.tp.0000132785.96091.d9, indexed in Pubmed: 15371686.

33. Nathan DP, Wang GJ, Woo EY, et al. Open and endovascular repair of hepatic artery aneurysm: two case reports and review of the literature. Vascular. 2011; 19(1): 42-46, doi: 10.1258/vasc.2010.cr0208, indexed in Pubmed: 21489926.

34. Olewnik $Ł$, Wysiadecki G, Polguj M, et al. Types of coeliac trunk branching including accessory hepatic arteries: a new point of view based on cadaveric study. Folia Morphol. 2017; 76(4): 660-667, doi: 10.5603/FM.a2017.0053, indexed in Pubmed: 28612916.

35. Pai RS, Hunnargi AS, Srinivasan M. Accessory left hepatic artery arising from common hepatic artery. Indian J Surg.
2008; 70(2): 80-82, doi: 10.1007/s12262-008-0021-0, indexed in Pubmed: 23133027.

36. Paloyo S, Nishida S, Fan Ji, et al. Portal vein arterialization using an accessory right hepatic artery in liver transplantation. Liver Transpl. 2013; 19(7): 773-775, doi: 10.1002/ It.23653, indexed in Pubmed: 23554089.

37. Panagouli E, Venieratos D. Right accessory hepatic artery arising from the left gastric artery: a case report. Rom J Morphol Embryol. 2011; 52(3 Suppl): 1143-1145, indexed in Pubmed: 22119839.

38. Paraskevas GK, Raikos A. Multiple aberrant coeliac trunk ramifications. Singapore Med J. 2011; 52(7): e147-e149, indexed in Pubmed: 21808947.

39. Polat $E$, Ozogul YB, Ercan M, et al. Management of hepatic artery aneurysms. Bratisl Lek Listy. 2012; 113(11): 676-679, doi: 10.4149/bll_2012_154, indexed in Pubmed: 23137209.

40. Polguj M, Gabryniak T, Topol M. The right accessory hepatic artery; a case report and review of the literature. Surg Radiol Anat. 2010; 32(2): 175-179, doi: 10.1007/s00276009-0536-5, indexed in Pubmed: 19669076.

41. Pulitano C, Joseph D, Sandroussi C, et al. Hepatic artery stenosis after liver transplantation: is endovascular treatment always necessary? Liver Transpl. 2015; 21(2): 162-168, doi: 10.1002/lt.24043, indexed in Pubmed: 25378262.

42. Serena TJ, Antypas E, Malay N, et al. Endovascular intervention of a mycotic pseudoaneurysm of accessory left hepatic artery arising from the left gastric artery presenting secondary to clostridium difficile colitis: a case report. Cureus. 2020; 12(4): e7802, doi: 10.7759/cureus.7802, indexed in Pubmed: 32461869.

43. Si-Tayeb K, Lemaigre FP, Duncan SA. Organogenesis and development of the liver. Dev Cell. 2010; 18(2): 175-189, doi: 10.1016/j.devcel.2010.01.011, indexed in Pubmed: 20159590.

44. Sureka B, Sharma N, Khera PS, et al. Hepatic vein variations in 500 patients: surgical and radiological significance. Br J Radiol. 2019; 92(1102): 20190487, doi: 10.1259/ bjr.20190487, indexed in Pubmed: 31271536.

45. Tigga SR, Saluja S, Budhiraja V, et al. Variant anatomy of the hepatic vasculature: importance in hepatobiliary resections. J Clin Diagn Res. 2017; 11(6): AD01-AD03, doi: 10.7860/ JCDR/2017/27193.10061, indexed in Pubmed: 28764144.

46. Wyżgowski P, Grzela T, Przybył M, et al. Rare case of hepatic artery pseudoaneurysm. Pol Przegl Chir. 2016; 88(3): 163-165, doi: 10.1515/pjs-2016-0046, indexed in Pubmed: 27428839.

47. Yamashita K, Hashimoto D, Itoyama R, et al. Accessory right hepatic artery branched from gastroduodenal artery. Surg Case Rep. 2015; 1: 90, doi: 10.1186/s40792-0150092-7, indexed in Pubmed: 26435909.

48. Yu YD, Kim DS, Byun GY, et al. Liver abscess developed after cadaveric liver transplantation due to ligation of an accessory right hepatic artery of the donor graft. J Korean Surg Soc. 2012; 83(4): 246-249, doi: 10.4174/ jkss.2012.83.4.246, indexed in Pubmed: 23091798. 